\title{
Caecal Herniation through the Foramen of Winslow: A Lesser Known Complication of the Lesser Sac
}

\author{
${ }^{1}$ Department of Radiology, Musgrove Park Hospital, Taunton, \\ United Kingdom \\ 2Department of Surgery, Musgrove Park Hospital, Taunton, \\ United Kingdom
}

Pia Charters ${ }^{1} \quad$ Andrew Lowe $^{1} \quad$ Richard Keogan ${ }^{1}$ Tom Edwards ${ }^{2}$

\begin{abstract}
Address for correspondence Pia Charters, MBChB, DFSRH, Department of Radiology, Musgrove Park Hospital, Parkfield Drive, Taunton, TA1 5DA, United Kingdom (e-mail: Pia.charters@nhs.net).
\end{abstract}
Abstract
Keywords
- cecum
- foramen of Winslow
- herniation
- laparoscopic surgery
- lesser sac

The foramen of Winslow is the omental communication between the greater and lesser sac. An internal hernia through the foramen of Winslow is extremely rare, accounting for $<0.1 \%$ of abdominal hernias and $8 \%$ of internal hernias. The majority of lesser sac hernias contain small bowel only, less commonly cecum or omentum. Diagnostic delay is associated with high morbidity and mortality, and accurate radiological interpretation is invaluable to guide surgical intervention. As such, the recognition of foramen of Winslow herniation on computed tomography (CT) is paramount. We present a case of caecal herniation that was recognized early and reversed within 48 hours of presentation, without the need for bowel resection.

\section{Introduction}

The foramen of Winslow is the omental communication between the greater and lesser sac. An internal hernia through the foramen of Winslow is extremely rare, accounting for $<0.1 \%$ of abdominal hernias and $8 \%$ of internal hernias. ${ }^{1}$ The majority of lesser sac hernias contain small bowel only, less commonly cecum or omentum. Diagnostic delay is associated with high morbidity and mortality and accurate radiological interpretation is invaluable to guide surgical intervention. ${ }^{2}$ As such, the recognition of foramen of Winslow herniation on computed tomography (CT) is paramount. We present a case of caecal herniation that was recognized early and reversed within 48 hours of presentation, without the need for bowel resection.

\section{Case Presentation}

A 72-year-old female presented by ambulance to the emergency department with a 3-day history of sudden onset abdominal pain and nausea. She was clinically diagnosed with biliary colic following a similar episode 2 weeks prior. The pain started in the epigastric region and radiated laterally across the abdomen, was exacerbated by eating and was associated with belching and reduced appetite. Her bowels, previously opening daily, had not opened for 72 hours and at presentation she was not passing flatus. Relevant past medical

received

May 8, 2019

accepted after revision

June 28, 2019

published online

November 5, 2019 history included total abdominal hysterectomy, borderline diet controlled type II diabetes, and paroxysmal atrial fibrillation managed with apixaban. There is a family history of gastric cancer on the paternal side. No recreational drug or alcohol use. On examination, her abdomen was soft with mild epigastric tenderness, negative Murphy's sign. Bowel sounds were reduced, no palpable masses, inguinal or femoral herniation. Digital rectal examination revealed an empty rectum. Clinical observations were within normal range.

Differential diagnoses included postprandial pain secondary to biliary colic and peptic ulcer disease.

Biochemical investigations revealed a mildly raised C-reactive protein. Hemoglobin, leukocytes, urea, electrolytes, liver function tests, and amylase were all within normal range. Arterial blood gas demonstrated a mild, uncompensated metabolic alkalosis with $\mathrm{pH} 7.46$ and $\mathrm{HCO}_{3}{ }^{-} 28.5$, normal lactate. The patient proceeded to have an abdominal ultrasound whereby the gallbladder was thoroughly examined and no evidence of calculi was visualized. The common bile duct was of normal caliber.

A CT abdomen/pelvis was subsequently performed. The patient was imaged on a Siemens Somatom Definition Flash 256 slice CT scanner manufactured by Siemens Healthineers AG, Forchheim, Germany. Axial $1.5 \mathrm{~mm}$ sections were performed through the abdomen and pelvis in the portovenous phase; 60 seconds postintravenous contrast injection using
License terms

Gastrointestinal and Abdominal Radiology

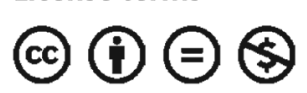


automated dosimetry at $100 \mathrm{kV}$ average $230 \mathrm{mAs} .200 \mathrm{~mL}$ intravenous Omnipaque 350 (iohexol) manufactured by GE Healthcare Ireland Limited, Cork, Ireland was delivered at the rate of $3 \mathrm{~mL} / \mathrm{s}$. SAFIRE (sinogram affirmed iterative reconstruction) raw data-based iterative reconstruction was performed.

The caecal pole was found to be ectopically situated, lying adjacent to the lesser curve of the stomach in the epigastric region suggestive of herniation through the foramen of Winslow into the lesser sac ( - Figs. 1 and 2A, B). The proximal ascending colon and terminal ileum had also passed through the epiploic foramen into the lesser sac (-Figs. 3 and $\mathbf{4}$ ). -Fig. 5 is a schematic anatomical representation of caecal herniation through the foramen of Winslow. No significant inflammatory changes were seen around the cecum and no abnormal gas seen in the caecal wall.

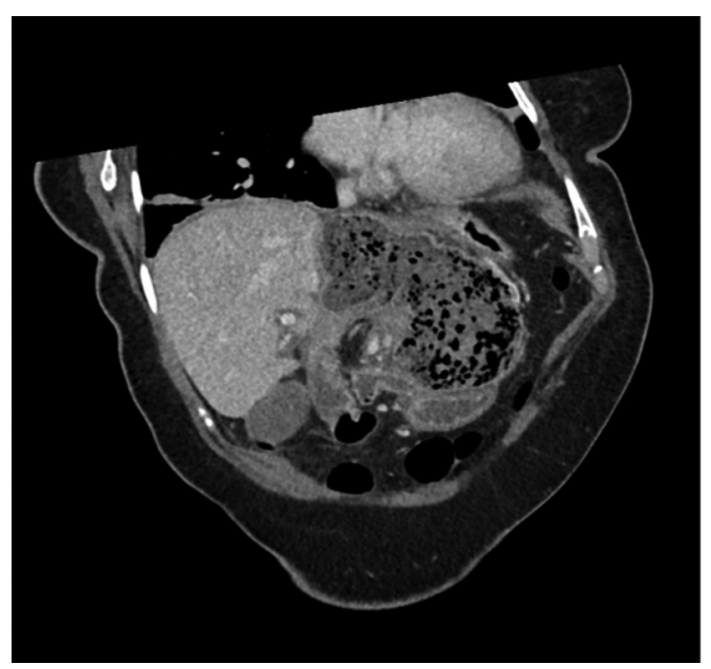

Fig. 1 Coronal oblique reconstruction of $1.5 \mathrm{~mm}$ abdominal slices imaged in the portovenous phase 60 seconds postintravenous iohexol contrast. The cecal pole is ectopically situated adjacent to the lesser curve of the stomach in the epigastric region suggestive of herniation into the lesser sac.
The patient was taken to theater the following day, intraoperative findings confirmed CT diagnosis that the terminal ileum and cecum had herniated through the foramen of Winslow. The aperture through which the cecum had herniated was so tight that laparoscopic reduction was not possible and the band was successfully reduced by open transverse laparotomy. The anterior border comprised the common bile duct (CBD), hepatic artery and hepatic vein, therefore the Hartmann's pouch of the gallbladder was sutured to the posterior abdominal wall following reduction. The right colon was completely free of omentum, therefore sutured to the right abdominal wall to keep it in place. The patient made a slow but steady recovery without complication and was discharged 9 days later.

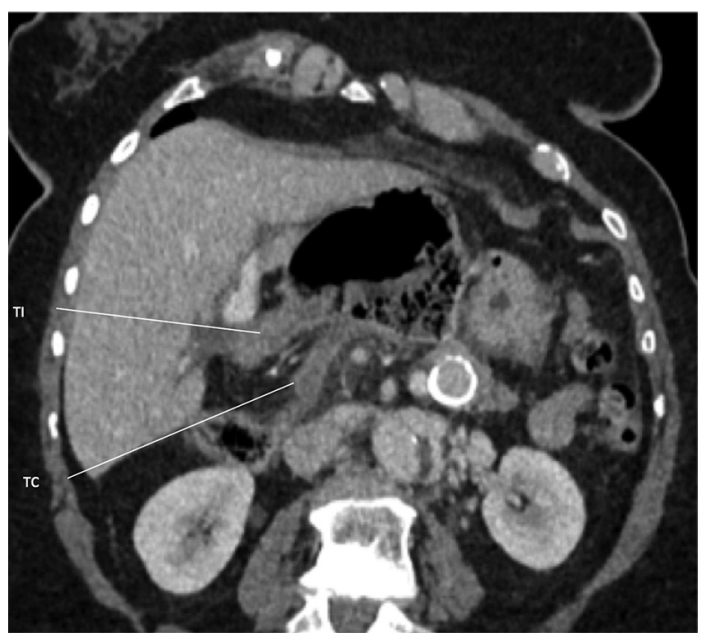

Fig. 3 Coronal oblique reconstruction of $1.5 \mathrm{~mm}$ abdominal slices imaged in the portovenous phase 60 seconds postintravenous iohexol contrast. TI, terminal ileum; TC, transverse colon.
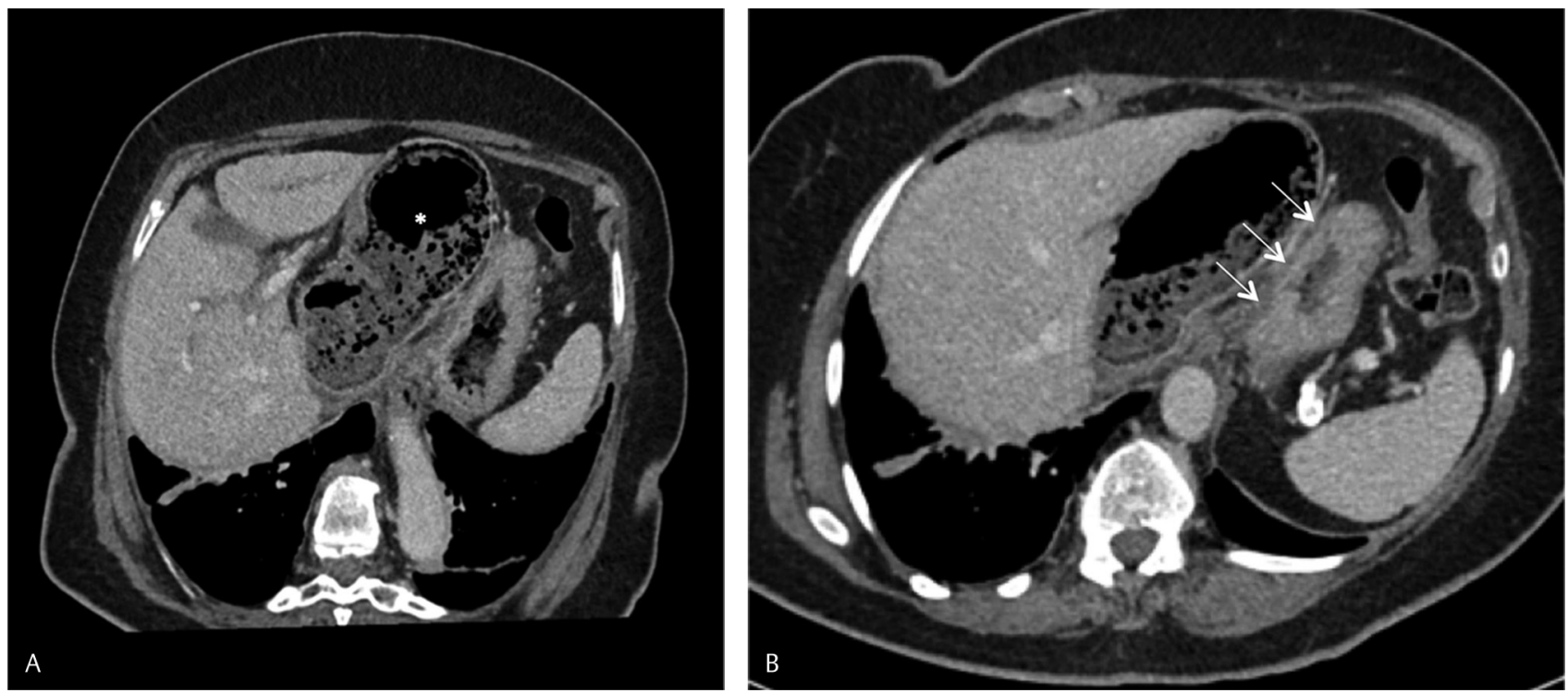

Fig. 2 (A) and (B) Axial oblique reconstructions demonstrating caecal, proximal ascending colon and terminal ileum herniation through the epiploic foramen into the lesser sac. Astrix, caecal pole; arrowheads, lesser curve of the stomach. 

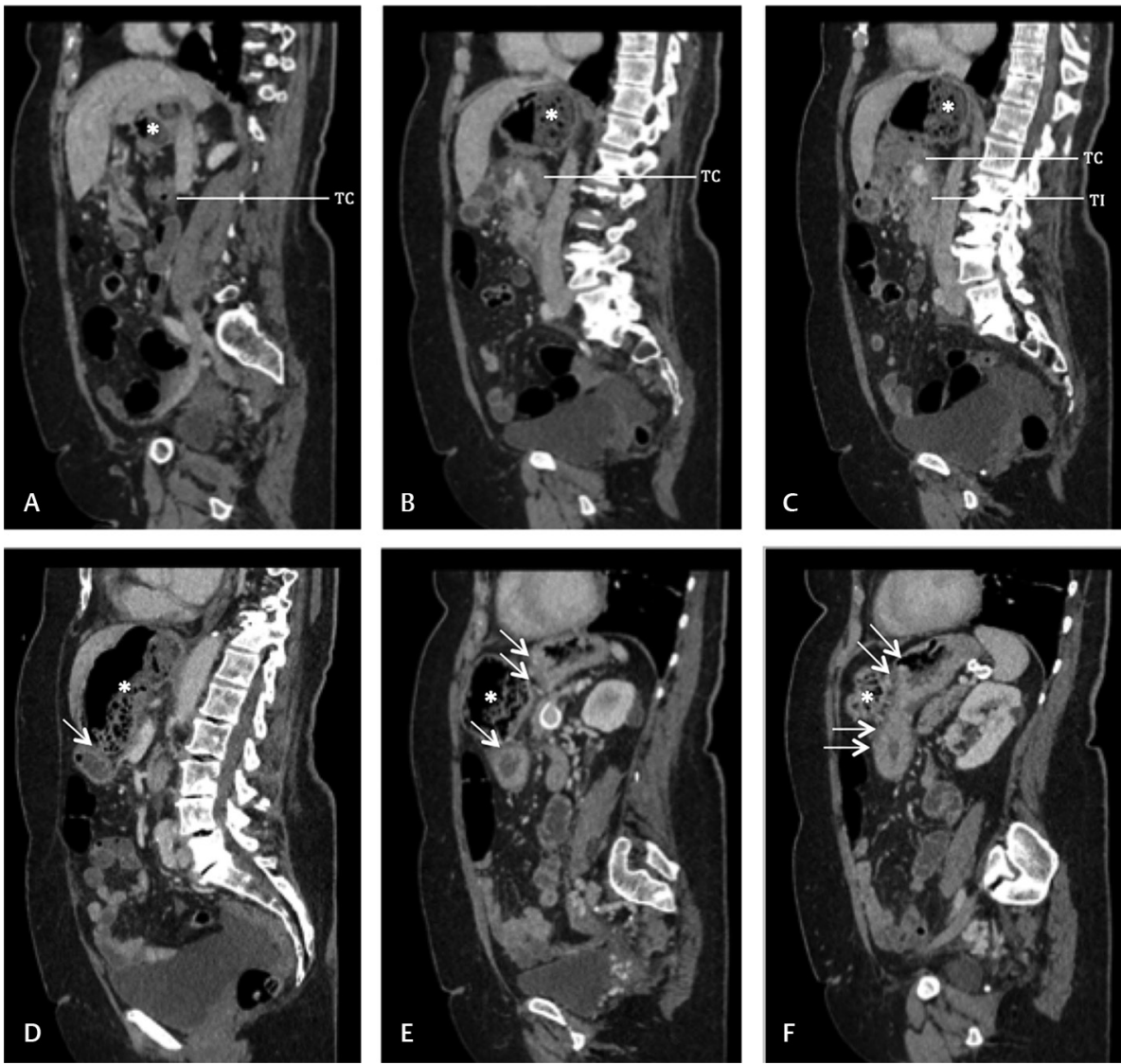

Fig. 4 (A-F) Sagittal reconstruction of $1.5 \mathrm{~mm}$ abdominal slices imaged in the portovenous phase 60 seconds postintravenous iohexol contrast. Images demonstrate caecal, terminal ileum, and proximal ascending colon herniation through the foramen of Winslow into the lesser sac.TC, transverse colon; TI, terminal ileum; asterix, caecal pole; arrowheads, lesser curve of the stomach.

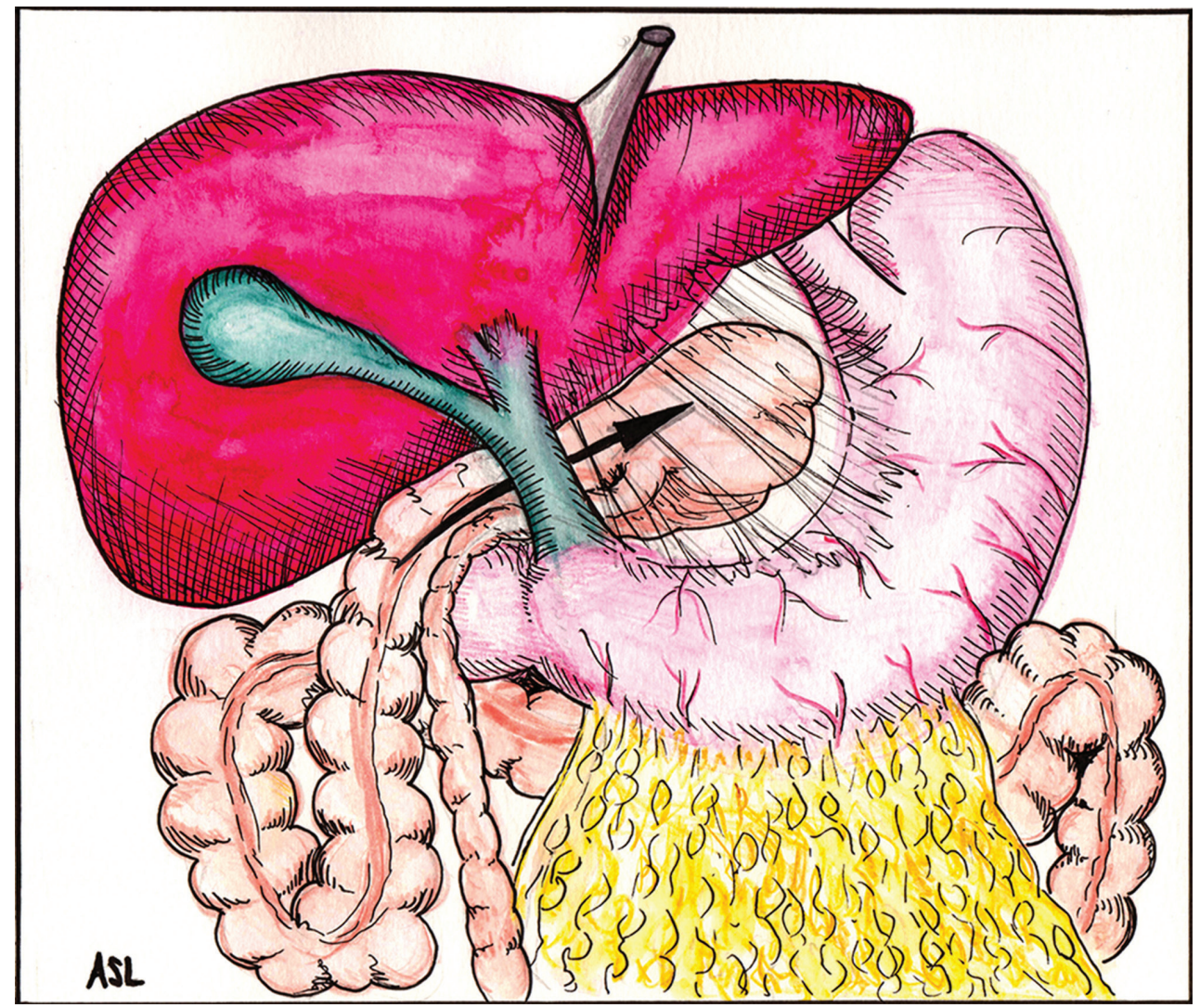

Fig. 5 Schematic representation of caecal herniation through the foramen of Winslow. Image Courtesy: Dr. Andrew Lowe. 


\section{Discussion}

The foramen of Winslow is the communication between the greater peritoneal cavity and the lesser sac. The posterior border is formed by the inferior vena cava and the anterior border by the hepatoduodenal ligament containing the CBD, hepatic artery and portal vein. Inferiorly lies the duodenum and superiorly the caudate lobe of the liver.

Herniation of the bowel through the foramen of Winslow is a rare condition, accounting for $<0.1 \%$ of abdominal herniation. ${ }^{1}$ The majority of cases contain small bowel only, rarely there have been reports of cecal, ascending and transverse colon, ${ }^{3}$ gallbladder, ${ }^{4}$ small bowel diverticulum ${ }^{5}$ or Meckel's diverticulum herniation through the lesser sac. ${ }^{6}$ Risk factors for lesser sac herniation are thought to include an enlarged foramen of Winslow, failed retroperitonealization of the right colon, a common intestinal mesentery or long small bowel mesentery. Other factors include atrophic greater omentum as in our case and sudden raised intra-abdominal pressure.

Presenting symptoms are nonspecific and can include both upper abdominal and chest pain. Prompt diagnosis is important because of the risk of necrosis to the herniated structures. Management involves urgent surgical reduction with bowel decompression and resection of nonviable tissue, frequently open surgery will be required. ${ }^{7.8}$

Historically, patients presenting with typical abdominal pain were investigated with explorative laparotomy, the diagnosis was made intraoperatively and often led to bowel resection because of ischemia. Such diagnostic delay therefore resulted in high morbidity and mortality. ${ }^{2}$ CT is fast and accessible allowing earlier diagnosis and earlier surgical intervention, even before the bowel becomes ischemic. Intervention ahead of bowel ischemia may possibly negate the need for bowel resection in this patient group with a projected reduction in morbidity and mortality.

\section{Learning Points}

- Internal herniation is a differential for abdominal pain without previous surgical intervention.
- Early investigation with CT is recommended if there is clinical suspicion of internal herniation or bowel ischemia.

- Herniation through the foramen of Winslow is a rare cause of bowel obstruction. Radiologists should be aware of its features on CT scan.

- An understanding of the relevant anatomy is crucial to reach radiological diagnosis and therefore guide early surgical intervention.

- Although caecal herniation through the lesser sac may be treated laparoscopically, frequently open surgery will be required.

\section{Conflict of Interest}

None declared.

\section{References}

1 Takeyama N, Gokan T, Ohgiya Y, et al. CT of internal hernias. Radiographics 2005;25(4):997-1015

2 Martin LC, Merkle EM, Thompson WM. Review of internal hernias: radiographic and clinical findings. AJR Am J Roentgenol 2006;186(3):703-717

3 Erskine JM. Hernia through the foramen of Winslow. a case report of the cecum incarcerated in the lesser omental cavity. Am J Surg 1967;114(6):941-947

4 MCCREA AN. Herniation of the gallbladder through the foramen of Winslow. Br J Surg 1951;38(151):386-387

5 Roberts PA. Hernia through the foramen of Winslow. Guys Hosp Rep 1953;102(3):253-264

6 Cullen TH. Herniation of an acutely inflamed Meckel's diverticulum through the foramen of Winslow. Arch Middx Hosp 1954;4(4):278-279

7 Tse G, Sollei T, Ali SM, Kukreja N. Caecal herniation through the foramen of Winslow. BJR Case Rep 2016;2(2):20150330

8 Harnsberger CR, McLemore EC, Broderick RC, et al. Foramen of Winslow hernia: a minimally invasive approach. Surg Endosc 2015;29(8):2385-2388 